\title{
Long-term creep behavior of the intervertebral disk: comparison between bioreactor data and numerical results
}

\author{
A. P. G. Castro ${ }^{1,2}$ *, C. P. L. Paul ${ }^{3,4}$, S. E. L. Detiger ${ }^{3,4,5}$, T. H. Smit ${ }^{3,4,5}$, B. J. van Royen ${ }^{3,4,5}$, J. C. Pimenta Claro', \\ M. G. Mullender ${ }^{3,4,6}$ and J. L. Alves ${ }^{1}$ \\ ' Center for Mechanical and Materials Technologies, Department of Mechanical Engineering, University of Minho, Guimarães, Portugal \\ 2 INSIGNEO Institute for in silico Medicine, Department of Mechanical Engineering, University of Sheffield, Sheffield, UK \\ ${ }^{3}$ Department of Orthopaedic Surgery, VU Medical Center, Amsterdam, Netherlands \\ ${ }^{4}$ Research Institute MOVE, Faculty of Human Movement Sciences, VU Medical Center, Amsterdam, Netherlands \\ ${ }^{5}$ Skeletal Tissue Engineering Group Amsterdam, VU Medical Center, Amsterdam, Netherlands \\ ${ }^{6}$ Department of Plastic, Reconstructive and Hand Surgery, VU Medical Center, Amsterdam, Netherlands
}

\section{Edited by:}

Fabio Galbusera, Ulm University, Germany

\section{Reviewed by:}

Henrique De Amorim Almeida, Polytechnic Institute of Leiria, Portugal Kyle Douglas Allen, University of Florida, USA

Andrea Malandrino, Massachusetts Institute of Technology, USA

*Correspondence:

A. P. G. Castro, Center for Mechanical and Materials Technologies, University of Minho, Campus de Azurem, Guimarães 4800-058, Portugal e-mail:apgcastro@dem.uminho.pt
The loaded disk culture system is an intervertebral disk (IVD)-oriented bioreactor developed by the VU Medical Center (VUmc, Amsterdam, The Netherlands), which has the capacity of maintaining up to 12 IVDs in culture, for approximately 3 weeks after extraction. Using this system, eight goat IVDs were provided with the essential nutrients and submitted to compression tests without losing their biomechanical and physiological properties, for 22 days. Based on previous reports (Paul et al., 2012, 2013; Detiger et al., 2013), four of these IVDs were kept in physiological condition (control) and the other four were previously injected with chondroitinase $A B C(C A B C)$, in order to promote degenerative disk disease (DDD). The loading profile intercalated $16 \mathrm{~h}$ of activity loading with $8 \mathrm{~h}$ of loading recovery to express the standard circadian variations. The displacement behavior of these eight IVDs along the first 2 days of the experiment was numerically reproduced, using an IVD osmo-poro-hyperviscoelastic and fiber-reinforced finite element (FE) model. The simulations were run on a custom FE solver (Castro et al., 2014). The analysis of the experimental results allowed concluding that the effect of the CABC injection was only significant in two of the four IVDs. The four control IVDs showed no signs of degeneration, as expected. In what concerns to the numerical simulations, the IVD FE model was able to reproduce the generic behavior of the two groups of goat IVDs (control and injected). However, some discrepancies were still noticed on the comparison between the injected IVDs and the numerical simulations, namely on the recovery periods. This may be justified by the complexity of the pathways for DDD, associated with the multiplicity of physiological responses to each direct or indirect stimulus. Nevertheless, one could conclude that ligaments, muscles, and IVD covering membranes could be added to the FE model, in order to improve its accuracy and properly describe the recovery periods.

Keywords: intervertebral disk, loaded disk culture system, custom finite element solver, creep behavior, circadian variations

\section{INTRODUCTION}

The intervertebral disk (IVD) is a highly inhomogeneous porous structure, which contains solid and fluid materials. It is, in its majority, avascular. The central structures of the disk, the nucleus pulposus (NP), and the annulus fibrosus (AF), are quite different in both constitution and function, but are paired structures, vertically limited by the cartilage (CEPs) and vertebral (VEPs) endplates (Raj, 2008; Shankar et al., 2009). The NP presents a gellike structure with embedded fibers and occupies the core of the IVD. Surrounding it, emerge an amount of concentrically arranged fibers supported on a porous matrix, which is the AF (Urban, 2000; Shankar et al., 2009; Hollingsworth and Wagner, 2011). The CEP is a layer of hyaline cartilage that is responsible for most of the nutrients exchange with the adjacent vertebral body (VB). One IVD and two VB constitute the motion segment (MS), which is the functional unit of the spine (Ebraheim et al., 2004; Raj, 2008; Schmidt and Reitmaier, 2013).

The correct identification of the IVD components and its healthiness has benefited from the advances on the medical imaging field. The IVDs are positioned in one of the most sensitive locations of the human body, next to the spinal canals and in close proximity to its nerve roots. Consequently, in vivo studies are quite challenging to perform (Nachemson and Morris, 1964). As a matter of fact, the work of Wilke et al. (1999) proved that those studies could be performed on humans, but they are highly dependent on the availability of volunteers, particularly because these experiments can be excessively painful and intrusive. Therefore, the major part of the information about the IVD behavior still comes from in vitro studies, using human or animal IVDs. These studies are usually performed ex vivo, but some up-to-date techniques 
allow the IVD to be kept alive after the sacrifice of the animal. The works of Chan et al. (2013), Gantenbein et al. (2006), Haglund et al. (2011), Korecki et al. (2007), Paul et al. (2012, 2013), and Walter et al. (2014) described bioreactors capable of providing nutrition and mechanical stimulation to the extracted IVD between 1 and 3 weeks. These versatile bioreactors are becoming the next benchmark for IVD experimental studies. Allied to these remarkable developments, numerical studies through finite element analysis (FEA) are benefiting from the improvements on the computational power to become more exhaustive and wide-ranging every day (Schmidt et al., 2013; Castro et al., 2014).

This work primarily deals with the analysis of the bioreactor data from the Department of Orthopedic Surgery of VUmc (Amsterdam, The Netherlands). The bioreactor system was developed within this group, and is denominated as loaded disk culture system (LDCS). In short, this system is capable of maintaining an IVD alive for at least 3 weeks once extracted, after the sacrifice of the animal, which is usually a goat. In comparison with the abovementioned analogous bioreactors, this is the one, which reports the longest period of IVD viability. The loading feature allows the IVD to be submitted to compression tests without losing its biomechanical and physiological properties (Paul et al., 2012, 2013). A schematic representation of this system is shown in Figure 1.

The LDCS reproduces the IVD native environment, by enabling the close monitoring of oxygen and nutrient supply levels, through the introduction of a culture medium, along with providing static or dynamic mechanical axial loading, as seen in Figure 1. The culture medium is pumped through a semi-porous titanium filter underneath the IVD at a speed of $3 \mathrm{ml} / \mathrm{h}$ and an osmolarity of 360-380 mOsm (Paul et al., 2012). The previous LDCS-related publications reported that, from the physiological point of view, a dynamic loading regime cause large dynamic displacement, while the static regimes induced a prolonged creep effect (Paul et al., 2012, 2013). Up to 12 IVDs may be simultaneously under experiment in this equipment.

This is a feasibility study, whose key goal is to numerically reproduce the LDCS experiments through FEA, using the custom

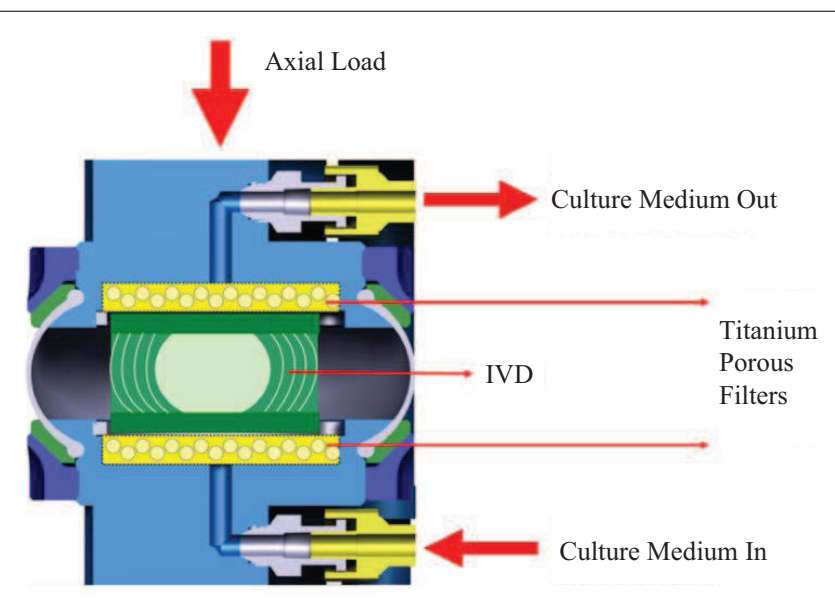

FIGURE 1 | Schematic representation of the LDCS. Adapted from Paul et al. (2012). tools developed on the Center for Mechanical and Materials Technologies of the University of Minho (CT2M-UM, Guimaraes, Portugal) (Alves et al., 2010; Castro et al., 2013, 2014; Cavalcanti et al., 2013).

\section{MATERIALS AND METHODS}

The object of study is an LDCS dataset, which contained the results of experiments performed with eight partial lumbar MS, i.e., one IVD, the two adjacent VEPs, and the connective membranes. In detail, four goats provided one control ("Cont") and one injected ("Inj") IVD each, i.e., one IVD was maintained intact during the lifetime of the animal (non-injected) and the adjacent IVD was injected with $0.5 \mathrm{U} / \mathrm{ml} 100 \mu \mathrm{L}$ of chondroitinase $\mathrm{ABC}(\mathrm{CABC})$ on $\mathrm{PBS}$ solution. The $\mathrm{CABC}$ is an enzyme that cleaves proteoglycans, i.e., this PBS-CABC compound is injected on the goat IVD with the aim of triggering degeneration (Detiger et al., 2013; Adams et al., 2014; Stefanakis et al., 2014). The lumbar IVDs used in the current study are derived from goats, which were also used in the study published by Detiger et al. (2013). Although the individual IVDs were not used for analyses in that study, the entire lumbar spinal segments of the goats were scanned with MRI pre-operatively and 12 months after $\mathrm{CABC}$ injection. This protocol intended to confirm degeneration of the IVDs due to CABC, using the Pfirmann scale (Pfirrmann et al., 2001; Griffith et al., 2007). Detiger and co-workers reported a large inter-individual variation for all parameters measured. However, no significant difference in Pfirmann scale was measured for the IVDs included in the current study (data not previously published).

The partial MS were tested under physiological loading conditions, which consisted of a sinusoidal load $(1 \mathrm{~Hz})$ of $150 \mathrm{~N}$ average and $100 \mathrm{~N}$ amplitude for $16 \mathrm{~h}$ (activity period), followed by other sinusoidal load $(1 \mathrm{~Hz})$ of $50 \mathrm{~N}$ average and $10 \mathrm{~N}$ amplitude for $8 \mathrm{~h}$ (resting period). Sinusoidal loadings are associated with dynamic loading regimes (Qasim et al., 2012). This loading profile is comparative to activities such as lying down and walking in goats and relaxed standing and non-supported sitting in humans. It must be highlighted that the transition between the activity and resting periods is performed with $1 \mathrm{~h}$ of triangular loading $(0.25 \mathrm{~Hz})$ of $200 \mathrm{~N}$ average and $100 \mathrm{~N}$ amplitude, which is also a dynamic loading regime (Hoogendoorn et al., 2008; Paul et al., 2012, 2013; Detiger et al., 2013).

The comparative finite element (FE) simulations were performed with the partial MS FE model shown in Figure 2, which included L3 and L4 VB (without facets) and the L3-L4 IVD. Ligaments and muscles were not considered, as they were also removed from the partial MS tested on the LDCS. The most relevant material properties of this model are summarized in Table 1, accordingly to the state-of-the-art of soft tissue and IVD constitutive modeling (Dreischarf et al., 2014; Freutel et al., 2014). For a more detailed description of the custom FE solver, the biphasic IVD constitutive modeling and MS FE model, the authors would like to refer to Castro et al. (2013, 2014). It must be highlighted that AF fibers' mechanical properties are assumed to evolve linearly through the axial plane, both in radial and circumferential directions (Eberlein et al., 2001), as previously described by Cavalcanti et al. (2013). Fiber angle also varies from $\pm 23.2^{\circ}$ at ventral position to $\pm 46.6^{\circ}$ at dorsal position, in accordance with Holzapfel et al. (2005). 
In order to compare the human IVD FE model with the LDCS data, some important assumptions had to be held, since the LDCS experiments were performed with goat IVDs (Schmidt and

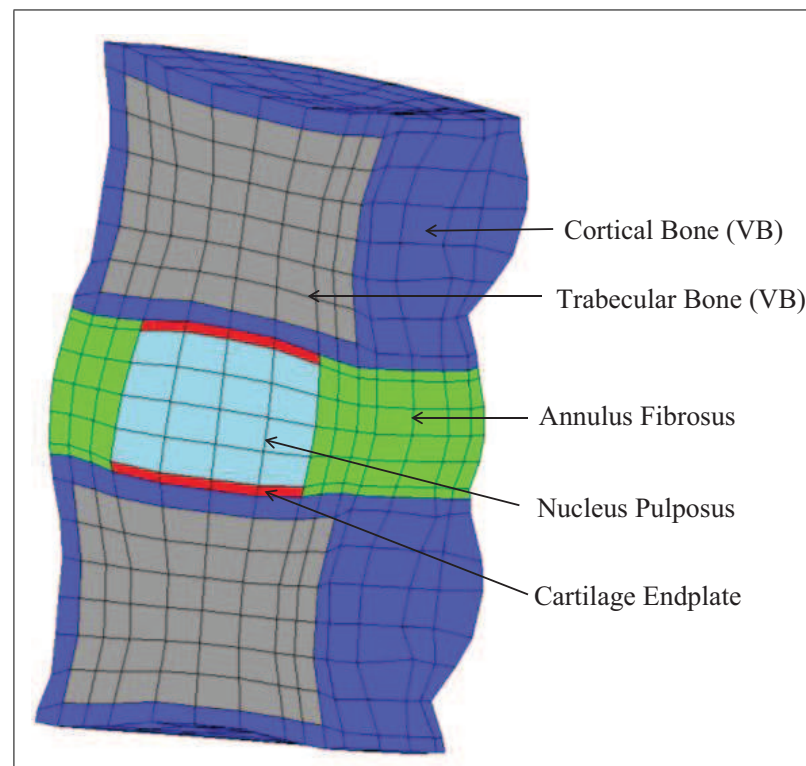

FIGURE 2 | Sagittal cut of the partial human L3-L4 MS FE model, which contains 1892 27-node quadratic hexahedral elements and 16,425 nodes.
Reitmaier, 2013; Reutlinger et al., 2014). The average axial cross section of the goat IVD is around $300-400 \mathrm{~mm}^{2}$ (Paul et al., 2012), accordingly to the experimental data, while the average axial cross section of the human IVD stands between 1200 and $1600 \mathrm{~mm}^{2}$. The IVD of the current FE model has an axial cross section of $1555.3 \mathrm{~mm}^{2}$ (Castro et al., 2014).

Consequently, the axial cross section of the human IVD is averagely four times larger than the goat IVD, so one could assume that the loads to be applied on the MS FE model should be four times greater than the loads experimentally applied on the goat IVDs, on the LDCS. In what concerns to the IVD height, the FE model has an average height of $12 \mathrm{~mm}$, while the goat IVDs have an average height of $9 \mathrm{~mm}$, which means that no normalization is needed. Previous studies verified that human and goat IVDs produce similar internal stresses, regardless of the geometric differences (Ayotte et al., 2001; Alini et al., 2008; Hoogendoorn et al., 2008; Schmidt and Reitmaier, 2013).

The other major simplification is related with the loading regimes. At the hourly time-scale, it was numerically verified that the sinusoidal loadings applied in the LDCS and the equivalent static average loadings produce comparable numerical outcome loadings. Since the sinusoidal wave meant additional computational effort, one adopted the lighter static and constant loadings.

Two daily cycles were simulated, after a preconditioning period of $8 \mathrm{~h}$, for osmotic equilibration before loading. The numerical physiological loading profile consists of $600 \mathrm{~N}$ for $16 \mathrm{~h}$ (activity period), followed by $200 \mathrm{~N}$ for $8 \mathrm{~h}$ (resting period). This model is known as "Native" (Galbusera et al., 2011; Paul et al., 2012). The

Table 1 | Material properties of the partial physiological MS FE model.

\begin{tabular}{|c|c|c|c|c|c|c|}
\hline & & NP & AF & CEP & TB & CB \\
\hline \multirow[t]{2}{*}{ Isotropy, Mooney Rivlin model (Schmidt et al., 2007) } & $C_{10}(\mathrm{MPa})$ & 0.15 & 0.18 & 1.00 & 41.67 & 3846.15 \\
\hline & $C_{01}(\mathrm{MPa})$ & 0.03 & 0.045 & 0.00 & 0.00 & 0.00 \\
\hline $\begin{array}{l}\text { Permeability, van der Voet model (Argoubi and Shirazi-Adl, } \\
\text { 1996; Ferguson et al., 2004) }\end{array}$ & M & 8.50 & 8.50 & 8.50 & 18.0 & 22.0 \\
\hline \multirow{4}{*}{$\begin{array}{l}\text { Viscoelasticity, Generalized Maxwell model (latridis et al., } \\
\text { 1997; Ehlers et al., 2009) }\end{array}$} & $a_{1}$ & 1.7 & - & & & \\
\hline & $\tau_{1}(\mathrm{~s})$ & 11.765 & - & & & \\
\hline & $a_{2}$ & 1.2 & - & & & \\
\hline & $\tau_{2}(\mathrm{~s})$ & 1.100 & - & & & \\
\hline \multirow{5}{*}{ Biphasic Swelling, Wilson model (Galbusera et al., 2011) } & $\phi_{\text {int }}$ & 0.83 & 0.83 & - & & \\
\hline & $\phi_{\text {ext }}$ & 0.92 & 0.92 & - & & \\
\hline & $C_{\text {ext }}\left(\mathrm{mmol} \mathrm{mm} \mathrm{mm}^{-3}\right)$ & 0.00015 & 0.00015 & - & & \\
\hline & $C_{\mathrm{F}, 0}\left(\mathrm{mmol} \mathrm{mm}^{-3}\right)$ & 0.00030 & 0.00018 & - & & \\
\hline & $n_{f, 0}$ & 0.80 & 0.70 & - & & \\
\hline
\end{tabular}

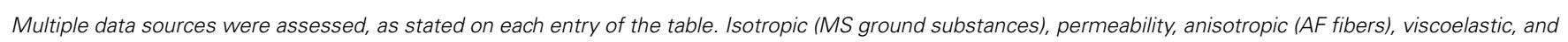
swelling properties were considered. 
transition between the activity and resting periods is performed with $800 \mathrm{~N}$ for $1 \mathrm{~h}$, in order to maintain a similar timeline with the experimental tests.

The effect of the PBS-CABC compound was numerically modeled through the reduction of the osmotic pressure gradient. As aforementioned, CABC cleaves proteoglycans, which are involved in the osmotic pressure mechanisms, through the regulation of the fixed charged density $\left(C_{\mathrm{F}}\right)$. In detail, proteoglycans have an essential role on fluid attraction and detainment, which allows the IVD to maintain its internal pressure, namely due to the "vesselwall"-like behavior of the AF fibers. Hence, if the proteoglycan content is reduced, the hydration of the IVD is also reduced and the osmotic pressure gradient is lower (Wognum et al., 2006; Massey et al., 2012; Stefanakis et al., 2014). The initial fixed charge density $\left(C_{F, 0}\right)$ of the NP was then reduced, in two levels, until it reached the initial fixed charge density of the AF, which remain unaltered (Table 1). This dual-step reduction is presented on Table 2. The model with the intermediate $C_{F, 0}$ reduction is entitled "Low OsmP 1 " and the model with the $C_{F, 0}$ value equal to the AF's properties is denominated "Low OsmP 2." Finally, in order to evaluate an extreme situation of proteoglycans cleavage, i.e., total split, a model without osmotic swelling was also tested ("No Swelling").

The other core hypothesis to simulate degeneration was the modification of the tissues' permeability, but this mechanism is highly dependent on the degeneration grade. Mild degeneration, as reported by Detiger et al. (2013), stands for grades III-IV on Thompson's scale (Thompson et al., 1990; Sivan et al., 2013). Low to mild degeneration are probably related to a decrease of CEP permeability, due to the calcification of this component, while mild to severe degeneration are more likely associated with an increase of CEP permeability, as a result of probable crack openings (Urban, 2000; Adams and Dolan, 2012; Stefanakis et al., 2014). The permeability variations on the degenerated NP and AF remain uncertain, but some studies pointed out that permeability could increase (Iatridis et al., 1998; Urban, 2000; Johannessen and Elliott, 2005; O'Connell et al., 2011). The proteoglycans might also be related to the permeability variations, but such association is also unclear. The IVD components are also described to increase in stiffness, as modeled by Natarajan et al. (2006) and Schmidt et al. (2007), but stiffness variations were not previously described in the literature as having a direct link to the injection of PBS-CABC compound (Detiger et al., 2013).

\section{RESULTS}

Figures 3 and 4 show the disk height variation (DHV) of the control and injected goat IVDs on the LDCS, respectively. The timeline was limited to two daily cycles. Other IVD-related outputs could

Table 2 | Osmotic swelling material properties and correspondent initial osmotic pressure of both native and reduced OsmP FE models.

\begin{tabular}{lcc}
\hline Model & NP $\boldsymbol{C}_{\boldsymbol{F}, \mathbf{0}}\left(\mathbf{m m o l ~} \mathbf{m m}^{\mathbf{- 3}}\right)$ & Initial NP OsmP (MPa) \\
\hline Native & 0.00030 & 0.189 \\
Low OsmP 1 & 0.00024 & 0.106 \\
Low OsmP 2 & 0.00018 & 0.036 \\
No Swelling & - & 0.000
\end{tabular}

be extracted from the FE solver, namely intradiscal pressure, fiber elongation of volume variation. Figure 5 shows the DHV along the test's timeline for the five numerical approaches, namely "Native," "Low OsmP 1," "Low OsmP 2," and "No Swelling" models. Finally, Figure 6 shows the comparison between the physiological DHV outcomes of the experimental and numerical tests ("Control" and "Native," respectively). Figure 7 shows the comparison between the injected IVDs DHV results of the experimental and the corresponding numerical tests, i.e., the two reduced OsmP models and the "No Swelling" model. Table 3 summarizes the DHV values of these seven DHV curves, demonstrating the differences between the four experimental tests, and also between the experimental tests and the numerical ones.

\section{DISCUSSION}

The control IVDs tend to maintain their height along the experiment, while the injected IVDs tend to lose height progressively (Figures 3 and 4, respectively). This systematic height reduction is mostly noticed on the loss of the ability to return to the same

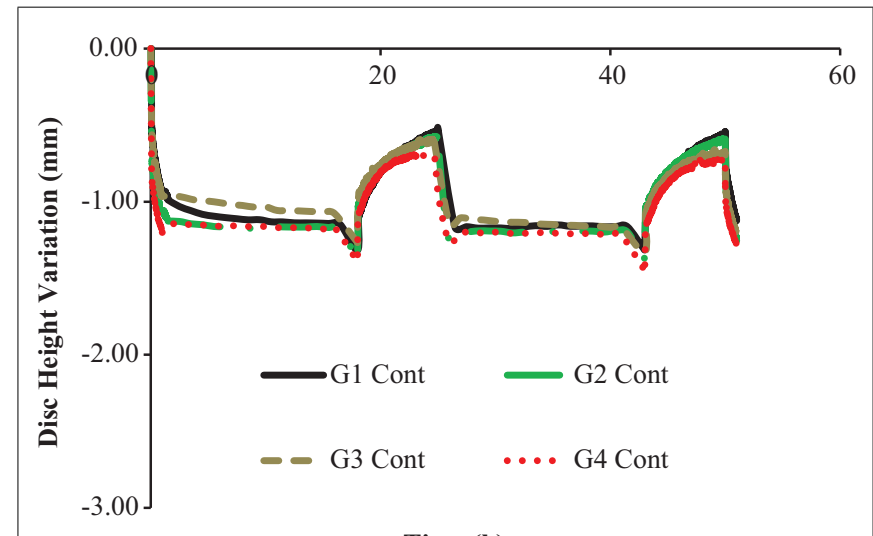

Time (h)

FIGURE 3 | Disk height variation outcomes of the LDCS, for two daily cycles, regarding the control goat IVDs.

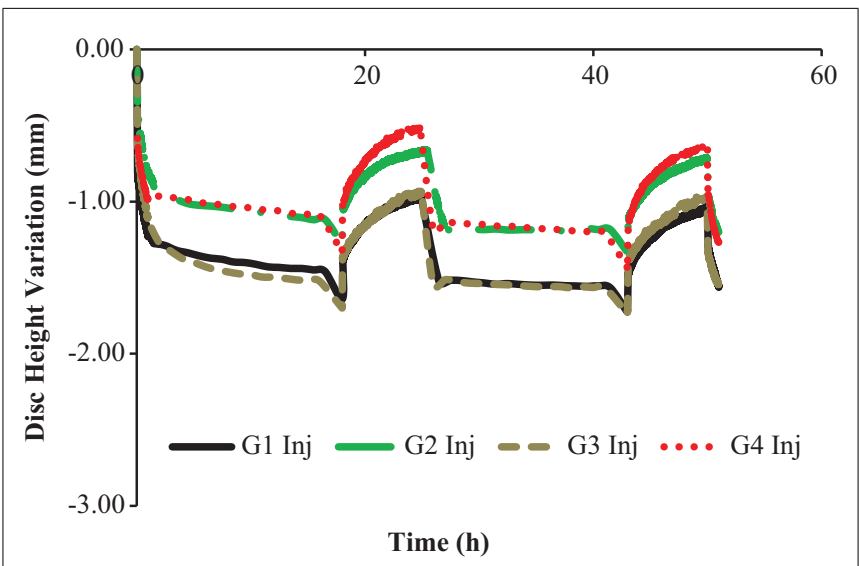

FIGURE 4 | Disk height variation outcomes of the LDCS, for two daily cycles, regarding the injected goat IVDs. 
height level after the recovery period. Such findings are in agreement with the work of Detiger et al. (2013), in which consistent evidence of IVD height reduction after the injection of the CABC

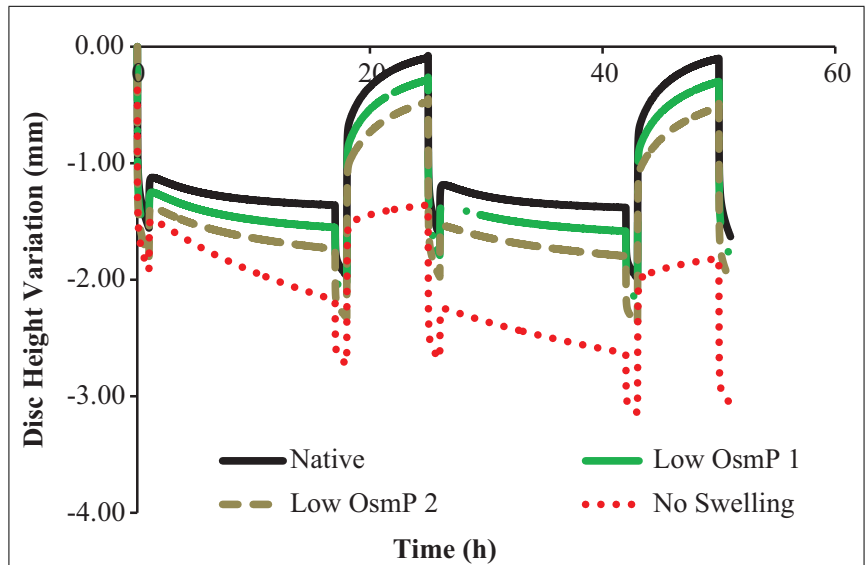

FIGURE 5 | Disk height variation outcomes of the four numerical approaches to the experimental data, namely "Native," “Low OsmP 1," "Low OsmP 2," and "No Swelling" models.

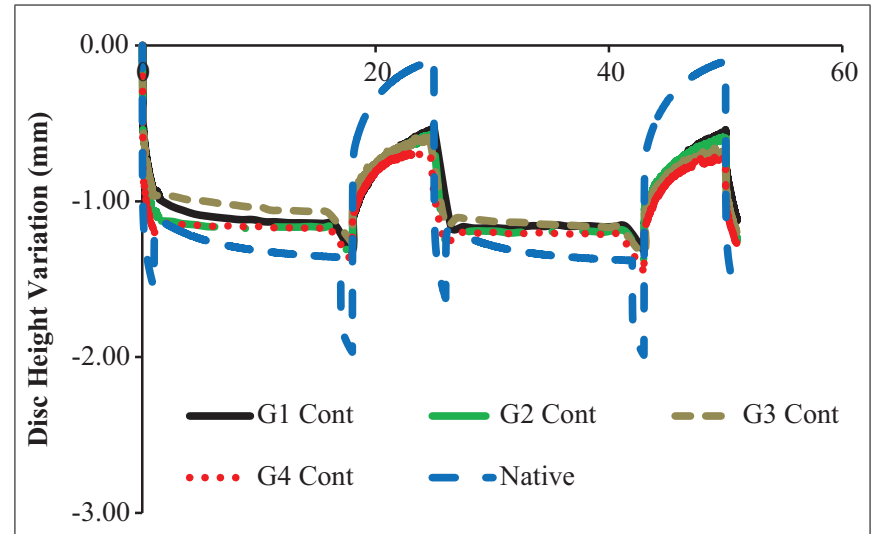

Time (h)

FIGURE 6 | Native DHV outcomes of the experimental and numerical tests, for two daily cycles, i.e., the four control goat IVDs are here compared with the native numerical model. compound was found. However, some discrepancies were noticed between the injected IVDs, which can be justified by the interspecimen variability of the goats. In fact, goats 1 and 3 seem to be more sensitive to the CABC compound than goats 2 and 4 .

In what concerns to Figure 5, it is noticeable that the behavior of the numerical model corresponds to what was theoretically expected. It must be highlighted that the transition periods are excluded from the current analysis of the maximum and minimum displacement measurements, as their purpose was just to establish the transition between the activity and recovery periods, on the experimental apparatus. Firstly, the "Native" model is able to fully recover the initial height on the resting periods, and the maximum DHV is inside the physiological displacement range. Secondly, the reduced OsmP models show some signs of degeneration, as the fluid flow seems to be diminished, and thus the initial height is not recovered. This effect is progressive, as the "Low OsmP 1 " model is less affected than the "Low OsmP 2" model. The maximum displacement also progressively increased (from the first to the second daily cycle), in comparison with the "Native" model. In addition, the "No Swelling" model shows clear signs of degeneration, as the maximum displacement is significantly increased and this increase is progressive throughout the daily cycles, without any sign of recovery. The recovery rate shall be understood as the

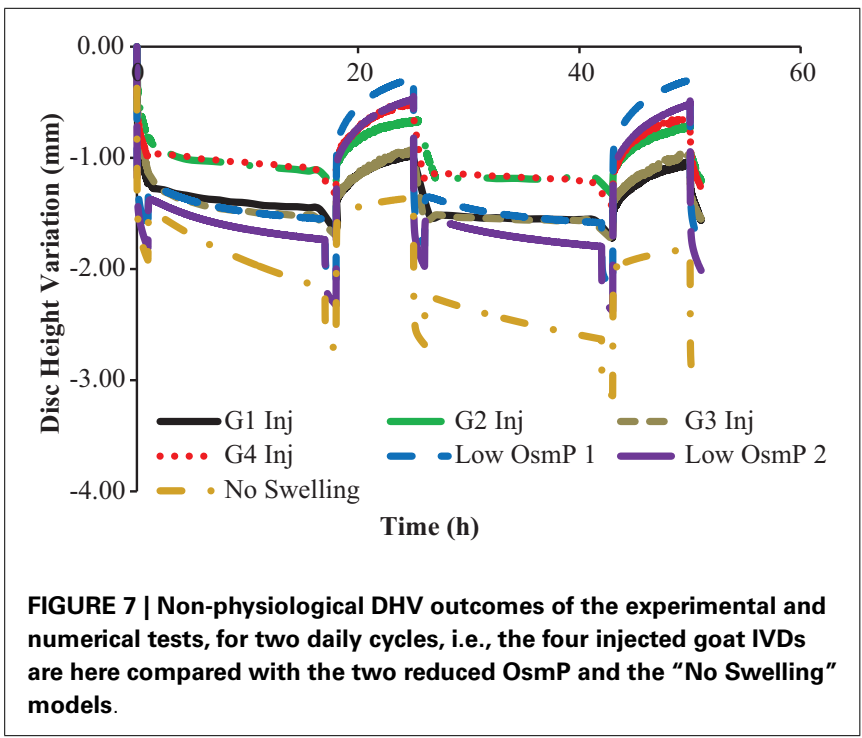

Table 3 | Summary of the DHV values of the four injected IVDs and the reduced OsmP models, after each activity and recovery period, obtained by numerical simulation.

IVD

$\mathrm{DHV}(\mathrm{mm})$

\begin{tabular}{llll}
\hline First activity period & First recovery period $\quad$ Second activity period $\quad$ Second recovery period
\end{tabular}

\begin{tabular}{llllll}
\hline Experimental & G1 inj & -1.46 & -0.96 & -1.57 & -1.18 \\
\\
& G2 inj & -1.12 & -0.68 & -1.56 & -0.72 \\
& G3 inj & -1.52 & -0.92 & -1.21 & -0.97 \\
Numerical & G4 inj & -1.09 & -0.49 & -1.59 & -0.63 \\
& Low OsmP 1 & -1.56 & -0.29 & -1.80 & -0.30 \\
& Low OsmP 2 & -1.74 & -0.47 & -2.63 & -1.82
\end{tabular}


difference between the DHV after the activity period and the DHV after the recovery period. The lowest recovery rate was registered by the "No Swelling" model, i.e., approximately $0.80 \mathrm{~mm}$ for the two daily cycles, meaning that this is the model with less capacity for regaining fluid content. At the light of the experimental tests, the behavior of the "No Swelling" model probably means that the proteoglycan cleavage promoted by the injected quantity of PBS$\mathrm{CABC}$ compound is only enough to cause mild degeneration, as expected. Considering the described outcomes, the situation of an IVD without any osmotic pressure gradient is certainly related to severe degeneration (Adams et al., 2014; Neidlinger-Wilke et al., 2014).

The comparison of the first two daily cycles of the native IVDs with the equivalent period of the "Native" model (Figure 6) shows that the numerical model is able to reproduce the physiological behavior of the goat IVD, particularly during the activity period. Numerically, the maximum DHV was $-1.36 \mathrm{~mm}$, while the average experimental measurement was $-1.20 \mathrm{~mm}$, considering the four native goat IVDs.

In what concerns to the resting periods, an important difference is noticed, as the numerical model is able to regain all the fluid lost during the activity period. The goat IVDs do not reach that DHV value of approximately $0.00 \mathrm{~mm}$, as their average DHV value on the resting period is $-0.58 \mathrm{~mm}$.

Nonetheless, these four control IVDs maintain the same DHV recovery level from the first to the second daily cycle, which was previously described as a sign of no degeneration. In other words, the DHV results indicate no degeneration, but incomplete recovery. This fact is probably related with the intrinsic behavioral differences between the goat and human IVDs, namely the specific biomechanical stimuli. The nutrition pathways included in the LDCS functioning and the ion-influenced fluid retention may also have a role in this situation. The numerical model helped to understand that the ideal situation is to fully recover the fluid on the resting periods. However, the action of AF fibers may also be limiting the range of $\mathrm{DHV}$, and this limit situation (maximum extension of the fibers) is not predicted in the FE solver. This action shall not be considerably different in human and goat spines.

Figure 7 shows that the "Low OsmP 1" model is closer to the injected IVDs from goats 1 and 3 than the "Low OsmP 2" model. The native IVDs presented a DHV difference of averagely $0.58 \mathrm{~mm}$ after the recovery periods, when compared with the "Native" model. The average difference between "G1 Inj" and "G3 Inj" and the "Low OsmP 1" model, after the recovery periods, is $0.50 \mathrm{~mm}$, i.e., the distance between these two injected IVDs and "Low OsmP 1" is close to what was measured for the native condition, which denotes consistency of the outcomes. In addition, the analogous difference for the maximum displacement is averagely $0.25 \mathrm{~mm}$, which is in agreement with the $0.16 \mathrm{~mm}$ difference found for the native condition. The DHV measurements from the injected IVDs of goats 2 and 4 may not be directly compared with these numerical outcomes, as their maximum displacement is significantly lower than the analogous measurement of the other two injected IVDs. The DHV measured after each recovery period is nearer to the numerical models, but the recovery rate is considerably uneven. Nevertheless, the average recovery rate of "G4 Inj" is inside the $0.50-0.60 \mathrm{~mm}$ range of the other two injected IVDs. The behavior of the "No Swelling" model is significantly distant from these injected IVDs.

On the numerical point of view, the results suggest that the MS FE model is excessively sensitive to the applied loads, having that the goat IVDs present a much more limited range of height variation. If these experimental tests were performed in vivo, one might question the amount of influence of the surrounding tissue (ligaments) on IVD mechanical properties and measurements. However, these structures were removed from the IVDs before the LDCS tests. On the one hand, one shall not neglect the influence of the external membranes covering each MS, since they also play a role on limiting the range of motion of IVD and other organs (Humphrey, 2003). On the other hand, previous studies from Ayotte et al. (2001) and Van der Veen et al. (2005) suggested that the fluid flow through VEP and CEP was modified once the IVD was extracted from the animal and/or tested isolated, which could cause the distorted recovery periods. Therefore, the behavioral differences are probably related to the intrinsic biochemical and biomechanical properties of the goat IVDs, including the action of the removed MS components.

On the experimental point of view, the injection of the PBSCABC compound on the goats caused degeneration on the targeted IVDs, but it could affect the adjacent levels similarly. The comparison with the MS FE model was successful, particularly for the physiological situations (control). The degenerated behavior of the IVD is extremely complex, given to the undisclosed physiological criteria related to the diverse possible loading and environmental stimuli. The degeneration prevention mechanisms are still to be fully understood (Adams and Roughley, 2006; Haschtmann et al., 2006; Qasim et al., 2012; Detiger et al., 2013; Galbusera et al., 2013; Adams et al., 2014; Benneker et al., 2014).

To sum up, this work substantiated that the LDCS is capable of mimicking the IVD in-body environment and also that the FE model is able to reproduce the experimental outcomes (control and injected). The successful association between these two distinct approaches to the IVD study allowed a very much detailed description of the proposed physiological and non-physiological situations.

In terms of future work, it would be interesting to simulate the full duration of the LDCS tests, i.e., up to 3 weeks of creep simulation with the IVD FE model. More computational resources would be needed to fulfill this task. In addition, ligaments, muscles, and IVD covering membranes would be an effective addition to the FE model, as their role is relevant for the global behavior of each MS.

\section{ACKNOWLEDGMENTS}

A. P. G. Castro, S. E. L. Detiger, T. H. Smit, J. C. Pimenta Claro and J. L. Alves would like to acknowledge the support of the European Project NP Mimetic (Biomimetic Nano-Fiber Based Nucleus Pulposus Regeneration for the Treatment of Degenerative Disk Disease), funded by the European Commission under FP7. A. P. G. Castro would also like to express his gratitude to Fundação para a Ciência e a Tecnologia (Portugal) for the Ph.D. grant SFRH/BD/63882/2009. 


\section{REFERENCES}

Adams, M. A., and Dolan, P. (2012). Intervertebral disc degeneration: evidence for two distinct phenotypes. J. Anat. 221, 497-506. doi:10.1111/j.1469-7580.2012. 01551.x

Adams, M. A., Lama, P., Zehra, U., and Dolan, P. (2014). Why do some intervertebral discs degenerate, when others (in the same spine) do not? Clin. Anat. doi:10.1002/ca.22404

Adams, M. A., and Roughley, P. J. (2006). What is intervertebral disc degeneration, and what causes it? Spine (Phila. Pa. 1976) 31, 2151-2161. doi:10.1097/01.brs. $0000231761.73859 .2 \mathrm{c}$

Alini, M., Eisenstein, S. M., Ito, K., Little, C., Kettler, A. A., Masuda, K., et al. (2008). Are animal models useful for studying human disc disorders/degeneration? Eur. Spine J. 17, 2-19. doi:10.1007/s00586-007-0414-y

Alves, J. L., Yamamura, N., Oda, T., and Teodosiu, C. (2010). "Numerical simulation of musculo-skeletal systems by V-Biomech," in CMBBE2010. eds J. Middleton, S. Evans, C. Holt, C. Jacobs, C. Atienza, and B. Walker (Valencia).

Argoubi, M., and Shirazi-Adl, A. (1996). Poroelastic creep response analysis of a lumbar motion segment in compression. J. Biomech. 29, 1331-1339. doi:10.1016/ 0021-9290(96)00035-8

Ayotte, D. C., Ito, K., and Tepic, S. (2001). Direction-dependent resistance to flow in the endplate of the intervertebral disc: an ex vivo study. J. Orthop. Res. 19, 1073-1077. doi:10.1016/S0736-0266(01)00038-9

Benneker, L. M., Andersson, G., Iatridis, J. C., Sakai, D., Härtl, R., Ito, K., et al. (2014). Cell therapy for intervertebral disc repair: advancing cell therapy from bench to clinics. Eur. Cell. Mater 27, 5-11.

Castro, A. P. G., Wilson, W., Huyghe, J. M., Ito, K., and Alves, J. L. (2013). "Validation of an open source finite element biphasic poroelastic model. Application to the intervertebral disc biomechanics," in Poromechanics V. eds C. Hellmich, B. Pichler, and D. Adam (Reston, VA: American Society of Civil Engineers), 2131-2139. doi:10.1061/9780784412992.251

Castro, A. P. G., Wilson, W., Huyghe, J. M., Ito, K., and Alves, J. L. (2014). Intervertebral disc creep behavior assessment through an open source finite element solver. J. Biomech. 47, 297-301. doi:10.1016/j.jbiomech.2013.10.014

Cavalcanti, C., Correia, H., Castro, A. P. G., and Alves, J. L. (2013). "Constituive modelling of the annulus fibrosus: numerical implementation and numerical analysis," in 2013 IEEE 3rd Portuguese Meeting in Bioengineering (ENBENG) (Braga, Portugal: IEEE), 1-4.

Chan, S. C. W., Walser, J., Käppeli, P., Shamsollahi, M. J., Ferguson, S., and Gantenbein-Ritter, B. (2013). Region specific response of intervertebral disc cells to complex dynamic loading: an organ culture study using a dynamic torsioncompression bioreactor. PLoS ONE 8:e72489. doi:10.1371/journal.pone.0072489

Detiger, S. E. L., Hoogendoorn, R. J. W., van der Veen, A. J., van Royen, B. J., Helder, M. N., Koenderink, G. H., et al. (2013). Biomechanical and rheological characterization of mild intervertebral disc degeneration in a large animal model. J. Orthop. Res. 31, 703-709. doi:10.1002/jor.22296

Dreischarf, M., Zander, T., Shirazi-Adl, A., Puttlitz, C. M., Adam, C. J., Chen, C. S., et al. (2014). Comparison of eight published static finite element models of the intact lumbar spine: predictive power of models improves when combined together. J. Biomech. 47, 1757-1766. doi:10.1016/j.jbiomech.2014.04.002

Eberlein, R., Holzapfel, G. A., and Schulze-Bauer, C. A. J. (2001). An anisotropic model for annulus tissue and enhanced finite element analyses of intact lumbar disc bodies. Comput. Methods Biomech. Biomed. Engin. 4, 209-229. doi:10.1080/10255840108908005

Ebraheim, N., Hassan, A., Lee, M., and Xu, R. (2004). Functional anatomy of the lumbar spine. Semin. Pain Med. 2, 131-137. doi:10.1016/j.spmd.2004.08.004

Ehlers, W., Karajan, N., and Markert, B. (2009). An extended biphasic model for charged hydrated tissues with application to the intervertebral disc. Biomech. Model. Mechanobiol. 8, 233-251. doi:10.1007/s10237-008-0129-y

Ferguson, S. J., Ito, K., and Nolte, L.-P. (2004). Fluid flow and convective transport of solutes within the intervertebral disc. J. Biomech. 37, 213-221. doi:10.1016/ S0021-9290(03)00250-1

Freutel, M., Schmidt, H., Dürselen, L., Ignatius, A., and Galbusera, F. (2014). Finite element modeling of soft tissues: material models, tissue interaction and challenges. Clin. Biomech. (Bristol, Avon) 29, 363-372. doi:10.1016/j.clinbiomech. 2014.01.006

Galbusera, F., Mietsch, A., Schmidt, H., and Wilke, H. (2013). Effect of intervertebral disc degeneration on disc cell viability: a numerical investigation. Comput. Methods Biomech. Biomed. Engin. 16, 328-337. doi:10.1080/10255842.2011.619184
Galbusera, F., Schmidt, H., Noailly, J., Malandrino, A., Lacroix, D., Wilke, H.-J., et al. (2011). Comparison of four methods to simulate swelling in poroelastic finite element models of intervertebral discs. J. Mech. Behav. Biomed. Mater. 4, 1234-1241. doi:10.1016/j.jmbbm.2011.04.008

Gantenbein, B., Grünhagen, T., Lee, C. R., van Donkelaar, C. C., Alini, M., and Ito, K. (2006). An in vitro organ culturing system for intervertebral disc explants with vertebral endplates: a feasibility study with ovine caudal discs. Spine (Phila. Pa. 1976) 31, 2665-2673. doi:10.1097/01.brs.0000244620.15386.df

Griffith, J. F., Wang, Y.-X. J., Antonio, G. E., Choi, K. C., Yu, A., Ahuja, A. T., et al. (2007). Modified Pfirrmann grading system for lumbar intervertebral disc degeneration. Spine (Phila. Pa. 1976) 32, E708-E712. doi:10.1097/BRS. 0b013e31815a59a0

Haglund, L., Moir, J., Beckman, L., Mulligan, K. R., Jim, B., Ouellet, J. A., et al. (2011). Development of a bioreactor for axially loaded intervertebral disc organ culture. Tissue Eng. Part C Methods 17, 1011-1019. doi:10.1089/ten.TEC.2011.0025

Haschtmann, D., Stoyanov, J. V., Ettinger, L., Nolte, L.-P., and Ferguson, S. J. (2006). Establishment of a novel intervertebral disc/endplate culture model: analysis of an ex vivo in vitro whole-organ rabbit culture system. Spine (Phila. Pa. 1976) 31, 2918-2925. doi:10.1097/01.brs.0000247954.69438.ae

Hollingsworth, N. T., and Wagner, D. R. (2011). Modeling shear behavior of the annulus fibrosus. J. Mech. Behav. Biomed. Mater. 4, 1103-1114. doi:10.1016/j. jmbbm.2011.03.019

Holzapfel, G. A., Schulze-Bauer, C. A. J., Feigl, G., and Regitnig, P. (2005). Single lamellar mechanics of the human lumbar annulus fibrosus. Biomech. Model. Mechanobiol. 3, 125-140. doi:10.1007/s10237-004-0053-8

Hoogendoorn, R. J. W., Helder, M. N., Kroeze, R. J., Bank, R. A., Smit, T. H., and Wuisman, P. I. J. M. (2008). Reproducible long-term disc degeneration in a large animal model. Spine (Phila. Pa. 1976) 33, 949-954. doi:10.1097/BRS. 0b013e31816c90f0

Humphrey, J. D. (2003). Review paper: continuum biomechanics of soft biological tissues. R. Soc. Lond. Proc. Ser. A Math. Phys. Eng. Sci. 459, 3-46. doi:10.1098/rspa.2002.1060

Iatridis, J., Setton, L., Foster, R. J., Rawlins, B., Weidenbaum, M., and Mow, V. C. (1998). Degeneration affects the anisotropic and nonlinear behaviors of human anulus fibrosus in compression. J. Biomech. 31, 535-544. doi:10.1016/S00219290(98)00046-3

Iatridis, J., Setton, L., Weidenbaum, M., and Mow, V. (1997). The viscoelastic behavior of the non-degenerate human lumbar nucleus pulposus in shear. J. Biomech. 30, 1005-1013. doi:10.1016/S0021-9290(97)00069-9

Johannessen, W., and Elliott, D. M. (2005). Effects of degeneration on the biphasic material properties of human nucleus pulposus in confined compression. Spine (Phila. Pa. 1976) 30, E724-E729. doi:10.1097/01.brs.0000192236.92867.15

Korecki, C. L., MacLean, J. J., and Iatridis, J. C. (2007). Characterization of an in vitro intervertebral disc organ culture system. Eur. Spine J. 16, 1029-1037. doi:10.1007/s00586-007-0327-9

Massey, C. J., van Donkelaar, C. C., Vresilovic, E., Zavaliangos, A., and Marcolongo, M. (2012). Effects of aging and degeneration on the human intervertebral disc during the diurnal cycle: a finite element study. J. Orthop. Res. 30, 122-128. doi: $10.1002 /$ jor. 21475

Nachemson, A., and Morris, J. M. (1964). In vivo measurements of intradiscal pressure. Discometry, a method for the determination of pressure in the lower lumbar discs. J. Bone Joint Surg. Am. 46, 1077-1092.

Natarajan, R. N., Williams, J. R., and Andersson, G. B. J. (2006). Modeling changes in intervertebral disc mechanics with degeneration. J. Bone Joint Surg. Am. 88(Suppl. 2), 36-40. doi:10.2106/JBJS.F.00002

Neidlinger-Wilke, C., Galbusera, F., Pratsinis, H., Mavrogonatou, E., Mietsch, A., Kletsas, D., et al. (2014). Mechanical loading of the intervertebral disc: from the macroscopic to the cellular level. Eur. Spine J. 23, S333-S343. doi:10.1007/ s00586-013-2855-9

O’Connell, G. D., Vresilovic, E. J., and Elliott, D. M. (2011). Human intervertebral disc internal strain in compression: the effect of disc region, loading position, and degeneration. J. Orthop. Res. 29, 547-555. doi:10.1002/jor.21232

Paul, C., Schoorl, T., Zuiderbaan, H., Zandieh Doulabi, B., van der Veen, A., van de Ven, P., et al. (2013). Dynamic and static overloading induce early degenerative processes in caprine lumbar intervertebral discs. PLoS ONE 8:e62411. doi:10.1371/journal.pone.0062411

Paul, C., Zuiderbaan, H. A., Zandieh Doulabi, B., van der Veen, A. J., van de Ven, P. M., Smit, T. H., et al. (2012). Simulated-physiological loading conditions 
preserve biological and mechanical properties of caprine lumbar intervertebral discs in ex vivo culture. PLoS ONE 7:e33147. doi:10.1371/journal.pone.0033147

Pfirrmann, C. W., Metzdorf, A., Zanetti, M., Hodler, J., and Boos, N. (2001). Magnetic resonance classification of lumbar intervertebral disc degeneration. Spine (Phila. Pa. 1976) 26, 1873-1878. doi:10.1097/00007632-200109010-00011

Qasim, M., Natarajan, R. N., An, H. S., and Andersson, G. B. J. (2012). Initiation and progression of mechanical damage in the intervertebral disc under cyclic loading using continuum damage mechanics methodology: a finite element study. J. Biomech. 45, 1934-1940. doi:10.1016/j.jbiomech.2012.05.022

Raj, P. (2008). Intervertebral disc: anatomy physiology pathophysiology treatment. Pain Pract. 8, 18-44. doi:10.1111/j.1533-2500.2007.00171.x

Reutlinger, C., Bürki, A., Brandejsky, V., Ebert, L., and Büchler, P. (2014). Specimen specific parameter identification of ovine lumbar intervertebral discs: on the influence of fibre-matrix and fibre-fibre shear interactions. J. Mech. Behav. Biomed. Mater. 30, 279-289. doi:10.1016/j.jmbbm.2013.11.019

Schmidt, H., Galbusera, F., Rohlmann, A., and Shirazi-Adl, A. (2013). What have we learned from finite element model studies of lumbar intervertebral discs in the past four decades? J. Biomech. 46, 2342-2355. doi:10.1016/j.jbiomech. 2013.07.014

Schmidt, H., Kettler, A., Rohlmann, A., Claes, L., and Wilke, H.-J. (2007). The risk of disc prolapses with complex loading in different degrees of disc degeneration - a finite element analysis. Clin. Biomech. (Bristol, Avon) 22, 988-998. doi:10.1016/j.clinbiomech.2007.07.008

Schmidt, H., and Reitmaier, S. (2013). Is the ovine intervertebral disc a small human one? A finite element model study. J. Mech. Behav. Biomed. Mater. 17, 229-241. doi:10.1016/j.jmbbm.2012.09.010

Shankar, H., Scarlett, J., and Abram, S. E. (2009). Anatomy and pathophysiology of intervertebral disc disease. Tech. Reg. Anesth. Pain Manag. 13, 67-75. doi:10.1053/j.trap.2009.05.001

Sivan, S. S., Merkher, Y., Wachtel, E., Urban, J. P. G., Lazary, A., and Maroudas, A. (2013). A needle micro-osmometer for determination of glycosaminoglycan concentration in excised nucleus pulposus tissue. Eur. Spine J. 22, 1765-1773. doi:10.1007/s00586-013-2714-8

Stefanakis, M., Luo, J., Pollintine, P., Dolan, P., and Adams, M. A. (2014). ISSLS Prize winner: mechanical influences in progressive intervertebral disc degeneration. Spine (Phila. Pa. 1976) 39, 1365-1372. doi:10.1097/BRS.0000000000000389

Thompson, J. P., Pearce, R. H., and Schechter, M. T., Adams, M. E., Tsang, I. K. Y., and Bishop, P. B. (1990). Preliminary evaluation of a scheme for grading the gross morphology of the human intervertebral disc. Spine (Phila. Pa. 1976) 15, 411-415. doi:10.1097/00007632-199005000-00012

Urban, J. P. G., (2000). The nucleus of the intervertebral disc from development to degeneration. Integr. Comp. Biol. 40, 53-61. doi:10.1093/icb/40.1.53

Van der Veen, A. J., Mullender, M., Smit, T. H., Kingma, I., and van Dieën, J. H. (2005). Flow-related mechanics of the intervertebral disc: the validity of an in vitro model. Spine (Phila. Pa. 1976) 30, E534-E539. doi:10.1097/01.brs.0000179306. 40309.3a

Walter, B. A., Illien-Jünger, S., Nasser, P. R., Hecht, A. C., and Iatridis, J. C. (2014). Development and validation of a bioreactor system for dynamic loading and mechanical characterization of whole human intervertebral discs in organ culture. J. Biomech. 47, 2095-2101. doi:10.1016/j.jbiomech.2014.03.015

Wilke, H.-J., Neef, P., Caimi, M., Hoogland, T., and Claes, L. E. (1999). New in vivo measurements of pressures in the intervertebral disc in daily life. Spine (Phila. Pa. 1976) 24, 755-762. doi:10.1097/00007632-199904150-00005

Wognum, S., Huyghe, J. M., and Baaijens, F. P. T. (2006). Influence of osmotic pressure changes on the opening of existing cracks in 2 intervertebral disc models. Spine (Phila. Pa. 1976) 31, 1783-1788. doi:10.1097/01.brs.0000227267.42924.bb

Conflict of Interest Statement: The authors declare that the research was conducted in the absence of any commercial or financial relationships that could be construed as a potential conflict of interest.

Received: 11 August 2014; accepted: 04 November 2014; published online: 20 November 2014.

Citation: Castro APG, Paul CPL, Detiger SEL, Smit TH, van Royen BJ, Pimenta Claro JC, Mullender MG and Alves JL (2014) Long-term creep behavior of the intervertebral disk: comparison between bioreactor data and numerical results. Front. Bioeng. Biotechnol. 2:56. doi: 10.3389/fbioe.2014.00056

This article was submitted to Biomechanics, a section of the journal Frontiers in Bioengineering and Biotechnology.

Copyright ( $) 2014$ Castro, Paul, Detiger, Smit, van Royen, Pimenta Claro, Mullender and Alves. This is an open-access article distributed under the terms of the Creative Commons Attribution License (CC BY). The use, distribution or reproduction in other forums is permitted, provided the original author(s) or licensor are credited and that the original publication in this journal is cited, in accordance with accepted academic practice. No use, distribution or reproduction is permitted which does not comply with these terms. 